\title{
Doxofylline is not just another theophylline!
}

This article was published in the following Dove Press journal: International Journal of COPD

\author{
Maria Gabriella Matera' \\ Clive Page ${ }^{2}$ \\ Mario Cazzola ${ }^{3}$ \\ 'Department of Experimental \\ Medicine, Unit of Pharmacology, \\ University of Campania 'Luigi \\ Vanvitelli', Naples, Italy; ${ }^{2}$ Sackler \\ Institute of Pulmonary Pharmacology, \\ Institute of Pharmaceutical Science, \\ King's College London, London, \\ UK; ${ }^{3}$ Department of Experimental \\ Medicine and Surgery, Chair of \\ Respiratory Medicine, University of \\ Rome 'Tor Vergata', Rome, Italy
}

\begin{abstract}
Doxofylline, which differs from theophylline in containing the dioxalane group at position 7 , has comparable efficacy to theophylline in the treatment of respiratory diseases, but with an improved tolerability profile and a favorable risk-to-benefit ratio. Furthermore, it does not have significant drug-drug interactions as exhibited with theophylline, which make using theophylline more challenging, especially in elderly patients with co-morbidities receiving multiple classes of drug. It is now clear that doxofylline also possesses a distinct pharmacological profile from theophylline (no significant effect on any of the known phosphodiesterase isoforms, no significant adenosine receptor antagonism, no direct effect on histone deacetylases, interaction with $\beta_{2}$-adrenoceptors) and therefore, should not be considered as just a modified theophylline. Randomized clinical trials of doxofylline to investigate the use of this drug to reduce exacerbations and hospitalizations due to asthma or COPD as an alternative to expensive biologics, and certainly as an alternative to theophylline are to be encouraged.
\end{abstract}

Keywords: doxofylline, theophylline, mechanisms of action, therapeutic effects, adverse effects

\section{Theophylline in the treatment of asthma and COPD}

Theophylline has been widely used to treat asthma and COPD since the 1930s, but while effective, it is a drug having a narrow therapeutic window and also many drugdrug interactions. ${ }^{1}$ Although the use of theophylline preparations is still defined in the Global Initiative for Asthma (GINA) 2015 report as add-on therapy for the treatment of adult patients with asthma, the increased availability of inhaled medicines with improved therapeutic windows means in reality less theophylline is being used. ${ }^{2}$ The Global Strategy for the Diagnosis, Management, and Prevention of COPD (GOLD) 2017 report also still includes theophylline in recognition of its bronchodilator effect in stable COPD, and because it has been demonstrated to elicit a further improvement in forced expiratory volume in $1 \mathrm{~s}$ and breathlessness when added to salmeterol. ${ }^{3}$ However, the evidence regarding the effect of low-dose theophylline on exacerbation rates is not clear and a recent meta-analysis of 7 observational studies suggests that theophylline slightly increases all-cause death in COPD patients. ${ }^{4}$ Again, with the increased availability of inhaled medicines with an improved safety profile, the current use of theophylline is declining for the treatment of COPD.

\section{The molecular mechanism(s) of action of theophylline}

The molecular mechanism(s) of action of theophylline is (are) not well understood, but several potential targets have been suggested, including non-selective inhibition of phosphodiesterases (PDE), inhibition of phosphoinositide 3-kinase- $\delta$ (PI3K- $\delta$ ), adenosine receptor antagonism and increased activity of certain histone deacetylases (HDACs) that deacetylate lysine residues in chromatin, thereby silencing gene transcription. ${ }^{5}$
Correspondence: Mario Cazzola Department of Experimental Medicine and Surgery, Chair of Respiratory Medicine, University of Rome 'Tor Vergata', Rome, Italy Emailmario.cazzola@uniroma2.it (c) (i) (\$) ๑ 2017 Matera et al. This work is published and licensed by Dove Medical Press Limited. The full terms of this license are avalable at https://www.dovepress.com/terms.php cc. hereby accept the Terms. Non-commercial uses of the work are permitted without any further permisision from Dove Medical Press Limited, provided the work is properly attributed. For permission for commercial use of this work, please see paragraphs 4.2 and 5 of our Terms (htpps//www.dovepress.com/terms.php). 
Theophylline relaxes airway smooth muscle (ASM) by inhibition mainly of PDE3 activity, and it has been suggested to prevent mediator release from a range of inflammatory cells by inhibition of PDE4 activity. ${ }^{6}$ However, the degree of inhibition is small at therapeutic concentrations and relatively high concentrations are needed to elicit effective PDE inhibitory activities. ${ }^{6}$ It is unlikely, therefore, that theophylline works as bronchodilator and anti-inflammatory drug solely through this mechanism. It has been suggested that the anti-inflammatory effects of theophylline may be mediated via activation of HDAC. ${ }^{7}$ HDAC counteracts the enzymatic activity of histone acetyltransferase that promotes histone acetylation and the exposure of gene promoter regions for transcription. ${ }^{8}$ These effects of theophylline are independent of PDE inhibition. ${ }^{7}$ Theophylline is also an antagonist of adenosine receptors with affinities against the human cloned adenosine receptors in the $\mathrm{mM}$ range, $\left(\mathrm{A}_{1}\right.$ receptor, $10-30 \mu \mathrm{M} ; \mathrm{A}_{2 \mathrm{~A}}$ receptor, $2-10 \mu \mathrm{M} ; \mathrm{A}_{2 \mathrm{~B}}$ receptor, 10-30 $\mu \mathrm{M}$; $\mathrm{A}_{3}$ receptor, 20-100 $\mu \mathrm{M}$ ), levels that can be achieved clinically. ${ }^{5}$ It has been proposed that antagonism of $A_{2 B}$ receptors for adenosine may account for the efficacy of this drug. ${ }^{9}$ However, antagonism of adenosine receptors has been reported to account for many of the side effects of theophylline, such as central nervous system (CNS) stimulation, cardiac arrhythmias (both via blockade of $\mathrm{A}_{1}$ receptors), gastric hypersecretion, gastroesophageal reflux, and diuresis ${ }^{10}$ and paradoxically, inhibition of adenosine $\mathrm{A}_{2 \mathrm{~A}}$ receptor signaling could potentially worsen inflammation. ${ }^{11}$

The documentation that low plasma levels of theophylline $(\sim 5 \mathrm{mg} / \mathrm{L})$ are able to enhance HDAC activity and restore the anti-inflammatory effects of corticosteroids in COPD by selectively inhibiting PI3K- $\delta^{12}$ is extremely interesting. This enzyme is a cell membrane localizing protein that leads to the subsequent phosphorylation of downstream signalling molecules (eg, Akt/PKB), which is activated by oxidative stress in lungs with COPD and involved in the inhibition of HDAC2 activity via phosphorylation. ${ }^{5}$ It has been suggested that the activation of HDAC 2 could contribute to the clinical effectiveness of theophylline as an anti-inflammatory drug and for its complementary activity to corticosteroids. In effect, in patients with COPD, a low dose of oral theophylline combined with an inhaled corticosteroid is more effective in reducing inflammation in sputum than either drug alone. ${ }^{13}$

\section{The development of other xanthines}

The numerous side effects associated with theophylline, drug-drug interactions and requirement for plasma monitoring limit the use of this drug. ${ }^{5}$ The propensity for these side effects are exacerbated in the elderly with comorbidities, impaired renal and liver function, in patients with cardiac failure and in patients on other medications that could give rise to drug-drug interactions, particularly if chronic overdosing occurs.

Nonetheless, the positive clinical effects of theophylline in airway disease, combined with its advantageous oral bioavailability, has spurred the development of other xanthines for the treatment of respiratory disease such as enprofylline, a $A_{2 B}$ selective receptor antagonist that showed some efficacy in the clinic, but was ultimately not developed due to several unwanted side effects, including headache and nausea/ vomiting and, mainly, abnormalities of liver function and variable blood levels despite constant oral dosage. ${ }^{14}$ Others have attempted to develop selective $\mathrm{A}_{1}$ receptor antagonists since this receptor type for adenosine is upregulated in subjects with asthma. ${ }^{15}$ Bamifylline, a known selective $A_{1}$ receptor antagonist is approved for the treatment of asthma in a number of countries. ${ }^{16}$ Also acebrofylline and doxofylline, and more selective PDE inhibitors such as roflumilast ${ }^{17}$ and RPL $554^{18}$, have been developed with the anticipation that such drugs would have greater efficacy than theophylline, but with an improved side effect profile. ${ }^{19}$ This review will discuss the state-of-the-art of one of these xanthines, doxofylline, and consider this in comparison with our current knowledge of theophylline.

\section{Doxofylline}

Doxofylline, chemically known as 7-(1,3-dioxolan-2ylmethyl)-3,7-dihydro-1,3 dimethyl-1H-purine-2,6-dione, is a xanthine derivative having both anti-inflammatory and bronchodilating activities. It differs from theophylline in containing the dioxalane group at position 7 (Figure 1). It has comparable efficacy with theophylline in the treatment

\section{Theophylline}
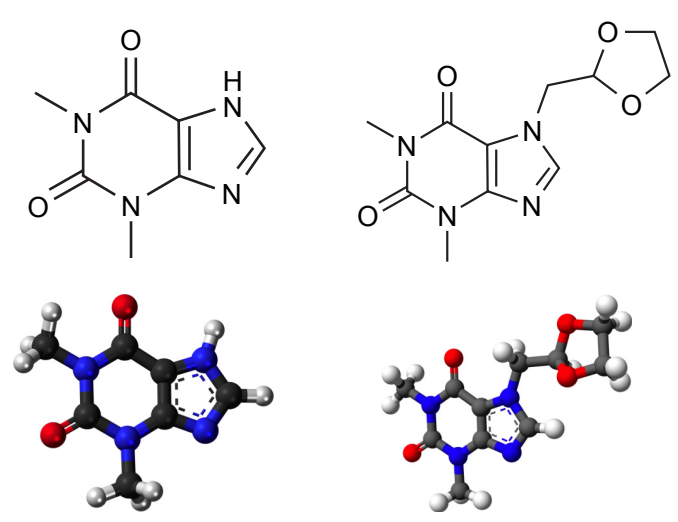

Figure I Bidimensional and tridimensional chemical structure images of theophylline and doxofylline. 
of respiratory diseases, but with an improved tolerability profile.

\section{The molecular mechanism(s) of action of doxofylline}

Doxofylline lacks significant adenosine receptor antagonism. Its affinities against the human, cloned adenosine $\mathrm{A}_{1}, \mathrm{~A}_{2 \mathrm{~A}}$ and $\mathrm{A}_{2 \mathrm{~B}}$ receptors are all higher than $100 \mu \mathrm{M} .{ }^{5}$ At concentrations that are likely to be achieved in patients following oral dosing, it only has a modest effect on adenosine $\mathrm{A}_{2 \mathrm{~A}}$ receptors, but not on any of the other known adenosine receptor subtypes. ${ }^{20}$ The decreased affinities toward adenosine $A_{1}$ and $A_{2}$ receptors, may contribute to its better safety profile.

Animal studies have shown that this poor adenosine antagonism is associated with a negligible stimulation of gastric secretion by doxofylline ${ }^{23}$ and importantly, the absence of significant cardiac effects. ${ }^{21}$ The cardiac activity of doxofylline in comparison with that of theophylline was investigated in guinea pig right and left atrial preparations, and in the anesthetized cat. In spontaneously beating right atria, doxofylline slightly increased the atrial rate, but only at $0.3 \mathrm{mM}$, while theophylline induced a concentrationdependent positive chronotropic effect that starts at $0.03 \mathrm{mM}$. In the anesthetized cat, heart rate increased by 13 beats $/ \mathrm{min}$ with $30 \mathrm{mg} / \mathrm{kg}$ doxofylline, but by 20 and 43 beats/min with 10 and $30 \mathrm{mg} / \mathrm{kg}$ of theophylline, respectively. ${ }^{24}$

Doxofylline also has no significant effect on any of the known PDE isoforms, except for $\mathrm{PDE}_{2 \mathrm{~A} 1}$, nor is its mechanism of action related to an effect on any of the known HDAC enzymes. $^{20}$

Recently, using nonlinear chromatography, frontal analysis and molecular docking, Zhang et $\mathrm{a}^{25}$ have documented that the interaction between doxofylline and $\beta_{2}$-adrenoceptors elicits relaxation of blood vessel and ASM. Ser ${ }^{169}$ and Ser ${ }^{173}$ seem to be the binding sites for the receptor-drug interaction and hydrogen bonding at these sites is likely to be the main driving force for this interaction. Apparently, the nitrogenatom of the imidazole ring and the oxygen atom of 1,3dioxolane contributed to the development of this hydrogen bonding. However, it has also been shown that doxofylline, similarly to theophylline, has no effect on formoterol-induced cAMP production (consistent with these drugs not really being significant PDE inhibitors at sensible concentrations) and does not augment formoterol-induced upregulation of the anti-inflammatory protein, mitogen-activated protein kinase phosphatase 1 (MKP-1), in ASM cells. ${ }^{26}$ Using human peripheral blood eosinophils isolated from asthma patients, Zhou et $\mathrm{al}^{27}$ documented that doxofylline could effectively decrease the open probability of the calcium-activated potassium channels as a result of both the shortening of the open period and the prolongation of the close time. Intriguingly, doxofylline differs from other methylxanthines in its inability to antagonize calcium-activated potassium channels known to be the sites for calcium channel blockers and thus does not interfere with the influx of calcium into cells, or mobilize intracellular calcium stores. ${ }^{28}$

There is evidence that doxofylline exerts anti-inflammatory activity as it is able to reduce the pleurisy induced by the inflammatory mediator platelet activating factor (PAF) in the rat. ${ }^{29}$ Additional preclinical studies have shown that doxofylline inhibits bacterial lipopolysaccharide (LPS)-induced neutrophil infiltration into the mouse lung. This effect was secondary to inhibiting leukocyte migration across vascular endothelial cells in vivo and in vitro, suggesting an important effect of this drug on leukocyte diapedesis. ${ }^{30}$ Furthermore, doxofylline administered for 3 months significantly reduced inflammatory changes and altered cell proliferation of the respiratory tract mucosa, such as infiltration of inflammatory cells, oedema and interstitial fibrosis, in a small group of patients suffering from chronic obstructive bronchitis. ${ }^{31}$ Interestingly, there is evidence that unlike theophylline, doxofylline does not inhibit tumor necrosis factor-induced interleukin (IL)-8 secretion in ASM cells. ${ }^{26}$

A very recent study has documented that doxofylline is able to exhibit corticosteroid sparing activity in two murine models of lung inflammation. ${ }^{32}$ The combination of doxofylline with dexamethasone at doses that themselves did not cause any significant reduction in the inflammation induced by LPS or allergen produced highly significant reductions in leukocyte infiltration into the lung in both models. Indeed the anti-inflammatory effect of the low dose dexamethasone in the presence of a low dose of doxofylline was equivalent to around a 10 times higher dose of dexamethasone administered alone. The precise mechanism of action of doxofylline to explain this corticosteroid sparing effect remains unknown but it is unlikely to be via an HDAC mediated mechanism. Doxofylline is also able to exert prophylactic effects against bronchoconstriction induced by $\mathrm{PAF}^{29}$ and methacholine in experimental animals. ${ }^{33}$ The results of a study that explored the effects of theophylline and doxofylline on airway responsiveness in beagles showed that doxofylline decreased airway responsiveness at a dose that did not affect heart rate and respiratory rate, ${ }^{33}$ which was not the case with theophylline under the same experimental conditions. ${ }^{33}$

\section{Pharmacokinetics of doxofylline}

In rats, orally administered doxofylline is rapidly absorbed, metabolized in the liver and partially excreted in the urine. ${ }^{34}$ It 
is equally distributed throughout the body, including the brain, although in much lower amounts than those absorbed. Three metabolites have been identified: hydroxyethyltheophylline $(\beta$-HET), the chief metabolite of doxofylline, and 2 isomers (cis and trans) of the sulfoxide, of which the trans-isomer predominates. The metabolites are also distributed in tissues, but do not accumulate. $\beta$-HET is a weak inhibitor of PDE activity and its affinity for adenosine $A_{1}, A_{2 A}$ and $A_{2 B}$ receptors is even lower than that of doxofylline. The oral toxicity of $\beta$-HET is about 3 times lower than that of doxofylline. ${ }^{35}$ Elimination is virtually complete at $24 \mathrm{~h}$.

At least in healthy humans, intravenous injection of doxofylline shows a biexponential serum concentration curve with a rapid elimination $\alpha$-phase of $<20 \mathrm{~min}$ and total clearance. ${ }^{36}$ This behavior suggests the involvement of an extra-renal component in its elimination. In Caucasian adults, after oral administration of $400 \mathrm{mg}$ twice daily for 5 days, the peak serum doxofylline concentration was found to be $15.21+1.73 \mu \mathrm{g} / \mathrm{mL}$ with a mean elimination half-life of $7.01+0.80 \mathrm{~h} .{ }^{37}$ A longer half-life results in effective plasma levels, also with twice daily dosing. Even after $12 \mathrm{~h}$ from the last oral dose, doxofylline was present in serum in appreciable concentrations. However, there was a large inter-subject variability in peak serum concentrations.

Ethnic differences in the pharmacokinetic profile of doxofylline have been reported. In healthy Chinese volunteers, the concentration time curve obtained from plasma drug concentration data fitted well to a first-order, 1-compartment open model. ${ }^{38}$ The drug was found to be rapidly absorbed with a marked individual variability, rapidly distributed in the body without an obvious distribution phase, and eliminated with variability among the individuals tested. However, in healthy Indian subjects, pharmacokinetic data were significantly different compared with the Chinese subjects. ${ }^{39}$ The issue of variability in the pharmacokinetics of doxofylline was also evident in 9 Korean volunteers, although there was no significant correlation between the doxofylline serum level and the body weight, creatinine clearance or age of the subjects. ${ }^{40}$

From a pharmacokinetic point of view, doxofylline importantly differs from theophylline also because it lacks the ability to interfere with the cytochrome enzymes CYP1A2, CYP2E1 and CYP3A4, thus preventing significant interaction with other drugs metabolized via these pathways in the liver. ${ }^{41}$ This is a major advantage of doxofylline over theophylline. Furthermore, doxofylline produces more stable serum concentrations than theophylline. Additionally, there is no evidence of an association between doxofylline levels and occurrence of adverse events. ${ }^{22}$ Therefore, there is no need for continued or repeated blood level monitoring with either low-dose or high-dose doxofylline, which is another big advantage of doxofylline over theophylline. ${ }^{19}$

\section{Therapeutic differences between doxofylline and theophylline}

A number of studies investigating the efficacy and safety of doxofylline have already been discussed in some previous reviews. ${ }^{19,42}$ Both articles concluded that doxofylline is an effective bronchodilator for relieving airway obstruction and displays a better safety profile with respect to theophylline, having a favorable risk-to-benefit ratio. Indeed, the number of patients needed to treat with doxofylline to spare 1 dropout due to theophylline was found to be $5 .{ }^{22}$

It is also noteworthy that in patients with endoscopicallyproven healed duodenal ulcers, doxofylline, unlike aminophylline, has a low secretagogue activity. ${ }^{43}$ It also has a superior gastric tolerability than theophylline. ${ }^{19}$ Furthermore, Sacco et $\mathrm{al}^{44}$ documented that the number of arousals per night when patients were treated with theophylline was almost double compared with when the subjects did not receive any medication, whereas doxofylline did not result in more arousals than no treatment. Sleep architecture and quality remained minimally affected by doxofylline, whereas it was substantially and significantly disrupted by theophylline.

Doxofylline does not increase myocardial oxygen demand, ${ }^{21}$ which is important when treating patients with ischemic heart disease, particularly relevant for patients with COPD since many such patients suffer from cardiovascular co-morbidities. Doxofylline is also unable to affect atrial frequency or the diastolic pressure in a significant way, unlike theophylline, which often causes hypotension.

In patients with chronic asthma, there is evidence that doxofylline $400 \mathrm{mg}$ t.i.d. is an effective treatment for relieving airway obstruction and displays a better safety profile with respect to theophylline $250 \mathrm{mg}$ t.i.d. with a favorable riskto-benefit ratio. ${ }^{22}$ More recently, this finding has also been documented in patients with mild bronchial asthma, whereby both theophylline $300 \mathrm{mg}$ twice a day and doxofylline $400 \mathrm{mg}$ twice a day improved lung function and symptoms, but where doxofylline had a better safety profile. ${ }^{45}$

Another study that enrolled patients suffering from asthma or COPD showed that doxofylline was more effective than theophylline as demonstrated by improvement in pulmonary function tests, as well as clinical symptoms, a reduced incidence of adverse effects and the need for 
Table I Comparison between doxofylline and theophylline

\begin{tabular}{ll}
\hline Doxofylline & Theophylline \\
\hline $\begin{array}{l}\text { No significant effect on any of the known PDE isoforms, } \\
\text { no significant adenosine receptor antagonism, no direct } \\
\text { effect on HDACs, interaction with } \beta_{2} \text {-adrenoceptors }\end{array}$ & $\begin{array}{l}\text { Non-selective inhibition of PDEs, inhibition of PI3K- } \delta \text {, adenosine receptor } \\
\text { antagonism and increased activity of certain HDACs }\end{array}$ \\
$\begin{array}{l}\text { No inhibition of TNF-induced IL-8 secretion in ASM cells } \\
\text { Low secretagogue activity }\end{array}$ & $\begin{array}{l}\text { Inhibition of TNF-induced IL-8 secretion in ASM cells } \\
\text { Increased gastric acid secretion and smooth muscle relaxation }\end{array}$ \\
$\begin{array}{l}\text { Sleep architecture and quality minimally affected probably } \\
\text { due to its lower affinity to the adenosine receptors }\end{array}$ & $\begin{array}{l}\text { Adverse cardiac effects caused by adenosine antagonism } \\
\text { Lack of interference with the cytochrome enzymes }\end{array}$ \\
$\begin{array}{l}\text { CYPIA2, CYP2EI and CYP3A4 } \\
\text { No known drug interactions }\end{array}$ & $\begin{array}{l}\text { Interference with the cytochrome enzymes CYPIA2, CYP2AI3, CYPIAI, CYP2EI, } \\
\text { CYP2D6 and CYP3A }\end{array}$ \\
Interactions with many drugs, including cimetidine, phenytoin, macrolides, \\
No known food interactions
\end{tabular}

Abbreviations: ASM, airway smooth muscle; IL, interleukin; HDACs, histone deacetylases; PDE, phosphodiesterase; TNF, tumor necrosis factor.

"rescue" bronchodilator use. ${ }^{46}$ The maximum beneficial effects of doxofylline were observed earlier in patients with asthma than those with COPD.

A trial conducted in patients with COPD presenting to the chest department of a medical college hospital in India showed that doxofylline $400 \mathrm{mg}$ twice a day was as effective as theophylline $400 \mathrm{mg}$ sustained release once a day. ${ }^{47}$ There was no statistically significant difference with respect to spirometric variables and symptom score in the 2 groups, and there was no significant difference in the 2 groups with respect to side effects.

However, another Indian study conducted in patients of COPD, that compared theophylline and doxofylline at doses recommended and commonly used in clinical practice, showed that both drugs significantly improved spirometric values and symptoms, cough, shortness of breath and nocturnal severity of symptoms. ${ }^{48}$ The main factor limiting the use of theophylline in this study was the high incidence of side effects, especially gastric distress (33\% in theophylline group and $15 \%$ in doxofylline group) and CNS stimulation.

A recent study that aimed to estimate the global cost related to the use of doxofylline and theophylline (associated drugs, specialist visits, hospital admissions, plasma drug monitoring), used data extracted from the Information System of the Pharmaceutical Prescriptions of the Marche Region in Italy for each ATC code (R03DA04 and R03DA11,) in the years 2008-2012.41 A total of 13,574 patients were treated with theophylline and 19,426 patients with doxofylline. The number of patients treated was $\sim 5,000$ per year. Co-prescription with other drugs, use of corticosteroids, mean number of visits and hospital admissions (per 100 patients) were all lower for doxofylline vs theophylline (1.55 vs 5.50, 0.3 vs $0.7,2.05$ vs 3.73 and 1.57 vs 3.3 ). The annual mean cost per patient was $€ 187.4$ for those treated with doxofylline and $€ 513.5$ for theophylline. This "real world" finding is really intriguing because the direct cost of doxofylline is higher than that of theophylline and demonstrates the pharmacoeconomic impact doxofylline can have at a population level when used regularly.

\section{Discussion}

The analysis of recent literature confirms that doxofylline produces clinical improvements comparable with those induced by theophylline but has a much better safety profile. However, it is now clear that doxofylline also possesses a distinct pharmacological profile from theophylline and therefore, should not be considered as just a modified theophylline (Table 1). Indeed, the improvement in the safety profile of doxofylline must be attributed to substantial differences in the pharmacological profile between this drug and theophylline.

Of particular importance is the observation that doxofylline does not have significant drug-drug interactions as exhibited with theophylline and which makes using theophylline more challenging, especially in elderly patients with co-morbidities receiving multiple classes of drug.

There are now a bewildering array of inhaled devices and formulations of drugs available for the treatment of asthma and COPD, which are often associated with poor adherence. ${ }^{49,50}$ Thus, the use of an orally active drug that is safe, effective and relatively inexpensive is to be encouraged, particularly for patients who find inhalers difficult to use or who do not get adequate control from other pharmacological classes. We would encourage further randomized clinical trials of doxofylline to investigate the use of this drug to reduce exacerbations and hospitalizations due to asthma or 
COPD as an alternative to expensive biologics, and certainly as an alternative to theophylline.

\section{Disclosure}

The authors are consultants at the ABC Farmaceutici (MGM and MC) and Eurodrug (CP) that manufacture and sell medicinal products containing doxofylline. The authors report no other conflicts of interest in this work.

\section{References}

1. Weinberger M, Hendeles L. Theophylline in asthma. $N$ Engl J Med. 1996;334(21):1380-1388.

2. Horak F, Doberer D, Eber E, et al. Diagnosis and management of asthma - Statement on the 2015 GINA Guidelines. Wien Klin Wochenschr. 2016;128(15-16):541-554.

3. Vogelmeier CF, Criner GJ, Martínez FJ, et al. Global strategy for the diagnosis, management, and prevention of chronic obstructive lung disease 2017 report: GOLD executive summary. Am J Respir Crit Care Med. 2017;195(5):557-582.

4. Horita N, Miyazawa N, Kojima R, Inoue M, Ishigatsubo Y, Kaneko T. Chronic use of theophylline and mortality in chronic obstructive pulmonary disease: a Meta-analysis. Arch Bronconeumol. 2016;52(5): 233-238.

5. Spina D, Page CP. Xanthines and phosphodiesterase inhibitors. Handb Exp Pharmacol. 2017;237:63-91.

6. Barnes PJ. Theophylline. Am J Respir Crit Care Med. 2013;188: 901-906.

7. Ito $\mathrm{K}, \mathrm{Lim} \mathrm{S}$, Caramori G, et al. A molecular mechanism of action of theophylline: Induction of histone deacetylase activity to decrease inflammatory gene expression. Proc Natl Acad Sci U S A. 2002;99: 8921-8926.

8. Urnov FD, Wolffe AP. Chromatin remodeling and transcriptional activation: the cast (in order of appearance). Oncogene. 2001;20: 2991-3006.

9. Chapman KR, Ljungholm K, Kallen A. Long-term xanthine therapy of asthma. Enprofylline and theophylline compared. Chest. 1994;106: 1407-1413.

10. Barnes PJ. Theophylline: new perspectives for an old drug. Am J Respir Crit Care Med. 2003;167:813-818.

11. Haskó G, Pacher P. $A_{2 A}$ receptors in inflammation and injury: lessons learned from transgenic animals. J Leukoc Biol. 2008;83(3):447-455.

12. To Y, Ito K, Kizawa Y, et al. Targeting phosphoinositide-3-kinasedelta with theophylline reverses corticosteroid insensitivity in chronic obstructive pulmonary disease. Am J Respir Crit Care Med. 2010;182: 897-904.

13. Ford PA, Durham AL, Russell RE, Gordon F, Adcock IM, Barnes PJ. Treatment effects of low dose theophylline combined with an inhaled corticosteroid in COPD. Chest. 2010;137:1338-1344.

14. Persson CG. Development of safer xanthine drugs for the treatment of obstructive airways disease. J Allergy Clin Immunol. 1986;78(4 Pt 2): $817-824$.

15. Fozard JR, Hannon JP. Adenosine receptor ligands: potential as therapeutic agents in asthma and COPD. Pulm Pharmacol Ther. 1999; 12(2):111-114.

16. Abbracchio MP, Cattabeni F. Selective activity of bamifylline on adenosine A1-receptors in rat brain. Pharmacol Res Commun. 1987; 19(8):537-545.

17. Boswell-Smith V, Page CP. Roflumilast: a phosphodiesterase-4 inhibitor for the treatment of respiratory disease. Expert Opin Investig Drugs. 2006; 15(9):1105-1113.

18. Calzetta L, Page CP, Spina D, et al. Effect of the mixed phosphodiesterase 3/4 inhibitor RPL554 on human isolated bronchial smooth muscle tone. J Pharmacol Exp Ther. 2013;346(3):414-423.
19. Page CP. Doxofylline: a "novofylline". Pulm Pharmacol Ther. 2010; 23(4):231-234.

20. van Mastbergen J, Jolas T, Allegra L, Page CP. The mechanism of action of doxofylline is unrelated to HDAC inhibition, PDE inhibition or adenosine receptor antagonism. Pulm Pharmacol Ther. 2012; 25(1):55-61.

21. Dini FL, Cogo R. Doxofylline: a new generation xanthine bronchodilator devoid of major cardiovascular adverse effects. Curr Med Res Opin. 2001;16(4):258-268.

22. Goldstein MF, Chervinsky P. Efficacy and safety of doxofylline compared to theophylline in chronic reversible asthma - a double-blind randomized placebo-controlled multicentre clinical trial. Med Sci Monit. 2002;8(4):CR297-CR304.

23. Franzone JS, Cirillo R, Barone D. Doxofylline and theophylline are xanthines with partly different mechanisms of action in animals. Drugs Exp Clin Res. 1988;14(7):479-489.

24. Cirillo R, Grossi E, Franzone JS. Doxofylline, an adenosine-nonblocking xanthine, does not induce cardiostimulant effects. Res Commun Chem Pathol Pharmacol. 1989;65(1):21-34.

25. Zhang Y, Zeng K, Wang J, Gao H, Nan Y, Zheng X. Identifying the antiasthmatic target of doxofylline using immobilized $\beta 2$-adrenoceptor based high-performance affinity chromatography and site-directed molecular docking. J Mol Recognit. 2016;29(10):492-498.

26. Patel BS, Kugel MJ, Baehring G, Ammit AJ. Doxofylline does not increase formoterol-induced cAMP nor MKP-1 expression in ASM cells resulting in lack of anti-inflammatory effect. Pulm Pharmacol Ther. 2017;45:34-39.

27. Zhou B, Cai SX, Zou F, Cai CQ, Zhao HJ. [Effect of doxofylline on calcium-activated potassium channels in human peripheral blood eosinophils in asthma]. Zhonghua Jie He He Hu Xi Za Zhi. 2005; 28(12):817-819. Chinese.

28. Franzone JS, Cirillo R, Reboani MC. Doxofylline differs from methylxanthines in its movement of cytosolic calcium. Int J Tissue React. 1991;13(3):131-138.

29. Franzone JS, Cirillo R, Biffignandi P. Doxofylline exerts a prophylactic effect against bronchoconstriction and pleurisy induced by PAF. Eur $J$ Pharmacol. 1989;165(2-3):269-277.

30. Riffo-Vasquez Y, Man F, Page CP. Doxofylline, a novofylline inhibits lung inflammation induced by lipopolysacharide in the mouse. Pulm Pharmacol Ther. 2014;27(2):170-178.

31. Cogo R, Castronuovo A. Effects of oral doxofylline on inflammatory changes and altered cell proliferation in chronic obstructive bronchitis. Eur Rev Med Pharmacol Sci. 2000;4:15-20.

32. Riffo-Vasquez Y, Venkatasamy R, Page CP. Steroid sparing effect of doxofylline. Pulm Pharmacol Ther. 2018;48:1-4.

33. Sugeta A, Imai T, Idaira S, Hirokoshi M, Okamoto M, Adachi M. [Effects of theophylline and doxofylline on airway responsiveness in dogs]. Arerugi. 1997;46(1):7-15. Japanese.

34. Cravanzola C, Grosa G, Franzone JS. Kinetic and metabolic studies of 7-(1,3-dithiolan-2-ylmethyl)-1,3-dimethylxanthine in the rat. Drugs Exp Clin Res. 1990;16(6):285-291.

35. Franzone JS, Cirillo R, Reboani MC. Pharmacological studies in animals of beta-hydroxyethyltheophylline, the major metabolite of doxofylline in humans. Methods Find Exp Clin Pharmacol. 1991;13(4):289-299.

36. Grossi E, Franzone IS. Farmacocinetica clinica della doxofillina. [Doxofillin Clinical Pharmacokinetics]. Farmaci. 1988;12:119-133. Italian.

37. Bologna $\mathrm{E}$, Laganà $\mathrm{A}$, Terracino $\mathrm{D}$, Bolignari $\mathrm{P}$, Biffignandi $\mathrm{P}$. Oral and intravenous pharmacokinetic profiles of doxofylline in patients with chronic bronchitis. J Int Med Res. 1990;18(4):282-288.

38. Jian G, Chunyan Z, Zhaolong C, Yuzhen L, Erzhang C. The pharmacokinetics of doxofylline in Chinese. Chin J New Drugs. 2000;6: 396-398.

39. Gannu R, Bandari S, Sudke SG, Rao YM, Shanka BP. Development and validation of a stability-indicating RP-HPLC method for analysis of doxofylline in human serum. Application of the method to a pharmacokinetic study. Acta Chromatographica. 2007;19:149-160. 
40. Lee JH, Namgung HW, Kwon SY, Yoon HI, Lee CT. The pharmacokinetic profiles of oral doxofylline and factors influencing the serum level [abstract]. Eur Respir J. 2011;38(Suppl 55):350s.

41. Mennini FS, Sciattella P, Marcellusi A, Marcobelli A, Russo A, Caputi AP. Treatment plan comparison in acute and chronic respiratory tract diseases: an observational study of doxophylline vs theophylline. Expert Rev Pharmacoecon Outcomes Res. 2017;17(5):503-510.

42. Shukla D, Chakraborty S, Singh S, Mishra B. Doxofylline: a promising methylxanthine derivative for the treatment of asthma and chronic obstructive pulmonary disease. Expert Opin Pharmacother. 2009;10(14): 2343-2356.

43. Lazzaroni M, Grossi E, Bianchi Porro G. The effect of intravenous doxofylline or aminophylline on gastric secretion in duodenal ulcer patients. Aliment Pharmacol Therap. 1990;4(6):643-649.

44. Sacco C, Barthiroli A, Gross E, Donner CF. The effects of doxofylline vs theophylline on sleep architecture in COPD patients. Monaldi Arch Chest Dis. 1995;50(2):98-103.
45. Margay SM, Farhat S, Kaur S, Teli HA. To study the efficacy and safety of doxophylline and theophylline in bronchial asthma. J Clin Diagn Res. 2015;9(4):FC05-FC08.

46. Lal D, Manocha S, Ray A, Vijayan VK, Kumar R. Comparative study of the efficacy and safety of theophylline and doxofylline in patients with bronchial asthma and chronic obstructive pulmonary disease. $J$ Basic Clin Physiol Pharmacol. 2015;26(5):443-451.

47. Akram MF, Nasiruddin M, Ahmad Z, Ali Khan R. Doxofylline and theophylline: a comparative clinical study. J Clin Diagn Res. 2012; 6(10):1681-1684.

48. Nagawaram PR, Kanchanpally V. Comparative study of theophylline and doxofylline in the treatment of stable chronic obstructive pulmonary disease. Int J Basic Clin Pharmacol. 2016;5(2):251-256.

49. Rogliani P, Calzetta L, Coppola A, et al. Optimizing drug delivery in COPD: the role of inhaler devices. Respir Med. 2017;124:6-14.

50. Rogliani P, Ora J, Puxeddu E, Matera MG, Cazzola M. Adherence to COPD treatment: Myth and reality. Respir Med. 2017;129:117-123.
International Journal of COPD

\section{Publish your work in this journal}

The International Journal of COPD is an international, peer-reviewed journal of therapeutics and pharmacology focusing on concise rapid reporting of clinical studies and reviews in COPD. Special focus is given to the pathophysiological processes underlying the disease, intervention programs, patient focused education, and self management protocols.

\section{Dovepress}

This journal is indexed on PubMed Central, MedLine and CAS. The manuscript management system is completely online and includes a very quick and fair peer-review system, which is all easy to use. Visit http://www.dovepress.com/testimonials.php to read real quotes from published authors.

Submit your manuscript here: http://www.dovepress.com/international-journal-of-chronic-obstructive-pulmonary-disease-journal 